\title{
Study the Sensitivity and Specificity of Urinary Arginase in Bladder Cancer Patients in Babylon Governorate
}

\author{
Frhan H. Kalaf ${ }^{1}$, Mufeed J. Ewadh ${ }^{2}$, Mohammad R.J. Abood ${ }^{2}$ \\ ${ }^{1}$ Lecturer, College of Medicine, University of Babylon, Iraq, \\ ${ }^{2}$ Prof., College of Medicine, University of Babylon, Iraq
}

\begin{abstract}
Background: Urinary Arginase is an enzyme found in the urine that can be measured by ELISA. The sensitivity and specificity of this test can also be checked.

Aim: The present study aims to determine Arginase in urine in the bladder cancer patient's the type of this study is case- controls study.

Method: The study was conducted during the period from August 2019 until February 2020. There are two groups, the first containing forty-five patients and the second forty-five control.

Results: The level of arginase in the urine of CA Bladder patients was evaluated in the two groups, the first group, G1 newly diagnosis (94.6+17.2), and second group G2 treatment and follow-up (.91.2 \pm 13.6 ), compared with normal patients control group (77.9 \pm 12.9$)$.In this study, urinary arginase was significantly higher in both groups of Bladder cancer patients G1 and G2 compared to normal control group of patients (CG) $\mathrm{P}$ value $<0.001$. In G1 (new diagnosis) urinary arginase was negligible in contrast with G2 (Treatment and fellow up) P-value $>0.05$. Throughout this analysis, the amount of urinary arginase increases with an increase in the cancer stage, thereby increasing cell degradation and releasing more protein molecules in the urine, such as arginase.
\end{abstract}

In Conclusion: This enzyme may serve as a useful biological urinary marker in bladder cancer while also being an indication of the progression of bladder cancer. the sensitivity of this test (92.59) and the Specificity (51.52)The sensitivity and specificity of this test should be improved in order to be used in the follow-up of patients with bladder cancer.

Keywords: Urinary Arginase, Bladder cancer, Specificity, Sensitivity.

\section{Introduction}

Bladder Cancer is the fourth most common cancer in males and the eighth in females and is the most common neoplasm of the urinary system ${ }^{[1]}$. Bladder cancer is a well-known condition that affects men more than

\footnotetext{
Corresponding Author:

Frhan H. kalaf

Lecturer, College of Medicine, University of Babylon, Iraq

e-mail: seedfrhan1965@gmail.com
}

women $^{[2]}$. The definition of urothelial carcinoma is the invasion of the basement membrane or lamina propria or deeper by neoplastic cells of urothelial origin It includes one of many types of malignant growth of the urinary bladder and It characterized by abnormal cells multiply without control in the bladder ${ }^{[3]}$. Cigarette smokling is the most well-established risk factor for Bladder cancer ${ }^{[4]}$. Bladder cancer (BC) is ones of the most common cancer worldwide. It is classified in muscle invasive (MIBC) and muscle non-invasive (NMIBC) $\mathrm{BC}^{[5]}$. NMIBCs frequently recur and progress to MIBCs with a reduced survival rate and frequent distant metastasis [6]. BC detection require unpleasant and expensive cystoscopy 
and biopsy, which are often accompanied by several adverse effects. Thus, there is an urgent need to develop novel diagnostic method for initial detection and surveillance in both MIBCs and NMIBCs ${ }^{[7]}$. Multiple urine-based tests approved by FDA for BC detection and surveillance are commercially available. However, at present, sensitivity, specificity and diagnostic accuracy of these urine-based assays are still suboptimal and, in the attend to improve them, novel molecular markers as well as multiple-assays must to be translated in clinic ${ }^{[8]}$. Arginase, a ubiquitous enzyme. It is present in yeasts, bacteria, plants, Invertebrates, and Vertebrates. It acts on the final step that leads to the production of urea (urea cycle) $)^{[9]}$. Arginine hydrolysis to form Ornithine and urea by the action of Arginase. Ornithine will convert to proline by ornithine aminotransferase (OAT) and polyamines by the ornithine decarboxylase (ODC) pathway ${ }^{[10]}$. Arginase is a manganese metalloenzyme that hydrolyses L-arginine to urea and L-ornithine. Arginase exists in two distinct isoforms, arginase I and II, that share $60 \%$ sequence homology ${ }^{[11]}$. Although both isoforms are found throughout the body, arginase I is a cytosolic enzyme mainly localized in the liver ${ }^{[12]}$. Hepatic arginase I contributes most of the body's total arginase activity and has a pivotal role in eliminating nitrogen formed during amino acid and nucleotide metabolism via the urea cycle ${ }^{[13]}$. More recently, arginase I expression has been demonstrated in extrahepatic tissues including endothelial cells and vascular smooth muscle cells ${ }^{[14]}$. Arginase II is a mitochondrial enzyme with a wide distribution and is expressed in the kidney, prostate, gastrointestinal tract, and the vasculature. The role of arginase II is not completely understood, but the enzyme is assumed to be involved in the regulation of $\mathrm{L}$-arginine homeostasis and production of L-ornithine for polyamine and proline synthesis for cell proliferation and development ${ }^{[15]}$. Both isoforms are expressed in the vasculature, but it appears as if the expression is both vessel and species dependent ${ }^{[16]}$. Increased arginase activity in mammals has been associated with cardiovascular and nervous system dysfunction and disease ${ }^{[17]}$. Arginase can be expressed in many various types of cells and can be stimulated by a vast variety of agents and conditions, depending on tissue and species ${ }^{[18]}$.

\section{Materials and Method}

The type of this study is case- controls study, which is done in the laboratory of Biochemistry Department, College of Medicine, University of Babylon. The study was conducted during the period from August 2019 until February 2020. The entire samples collected from patients attending Al-Hila Teaching Hospital, Urology Department at Margean Hospital Department of Oncology. The patient's group who subjected to this study were 45 patients divided into two groups depend on pathological staging: Group One (G1). Patients with this group newly diagnosis of CA bladder. This group including 19 patients in the range of age 35-76 years it is have 14 males (mean \pm standard deviation $59.7 \pm$ 10.7 years) and 5 females (mean \pm standard deviation $61,0 \pm 14.4$ years). Group Tow (G2) This group include patients with treatment of CA bladder and Follow up after treatments. This group including 26 patients in the range of age 52-86 years it is have 20 males (mean \pm standard deviation $65.4 \pm 7.7$ years) and 6 females (mean \pm standard deviation $65.4 \pm 12.1$ years). Anther grouped this includes forty- five apparently healthy subjects with an age range 31-67 years there are 11 females and 34 males. None of these subjects had a history of abnormal liver function, blood dyscrasia, hematuria and with any Lwoer part of urinary infection. Ten to twenty milliliters of urine were collected from patients and healthy persons to the urine cup and then collected in the plane tube were centrifuged at 4000xg for approximately 15 minutes. The supernatant was divided in two parts of 1.ml and transferred to the Eppendrof tube and stocked at $-20 \mathrm{C}$ until time of use. This Arginase enzyme-linked immunosorbent assay applies a technique called a quantitative sandwich immunoassay. The microtiter plate provided in this kit has been pre-coated with monoclonal antibody specific for Arg. Standards or samples are then added to the microtiter plate wells and Arg if present, will bind to the antibody precoated wells. In order to quantitatively determine the amount of Arg present in the sample, a standardized preparation of horseradish peroxidase (HRP)-conjugated polyclonal antibody, specific for Arg are added to each well to sandwichl the Arg immobilized on the plate. The microtiter plate undergoes incubation, and then the wells are thoroughly washed to remove all unbound components. Next, A and $\mathrm{B}$ substrate solution is added to each well. The enzyme (HRP) and substrate are allowed to react over a short incubation period. Only those wells that contain Arg and enzyme-conjugated antibody will exhibit a change in color. The enzyme-substrate reaction is terminated by the addition of a sulphuric acid solution and the color change is measured spectrophotometrically at a wavelength of $450 \mathrm{~nm}^{[19]}$. 


\section{Results and Discussion}

The mean \pm SD age of Bladder cancer patients were (60.6 \pm 10 years). age distributions of CA Bladder patients were as follow:

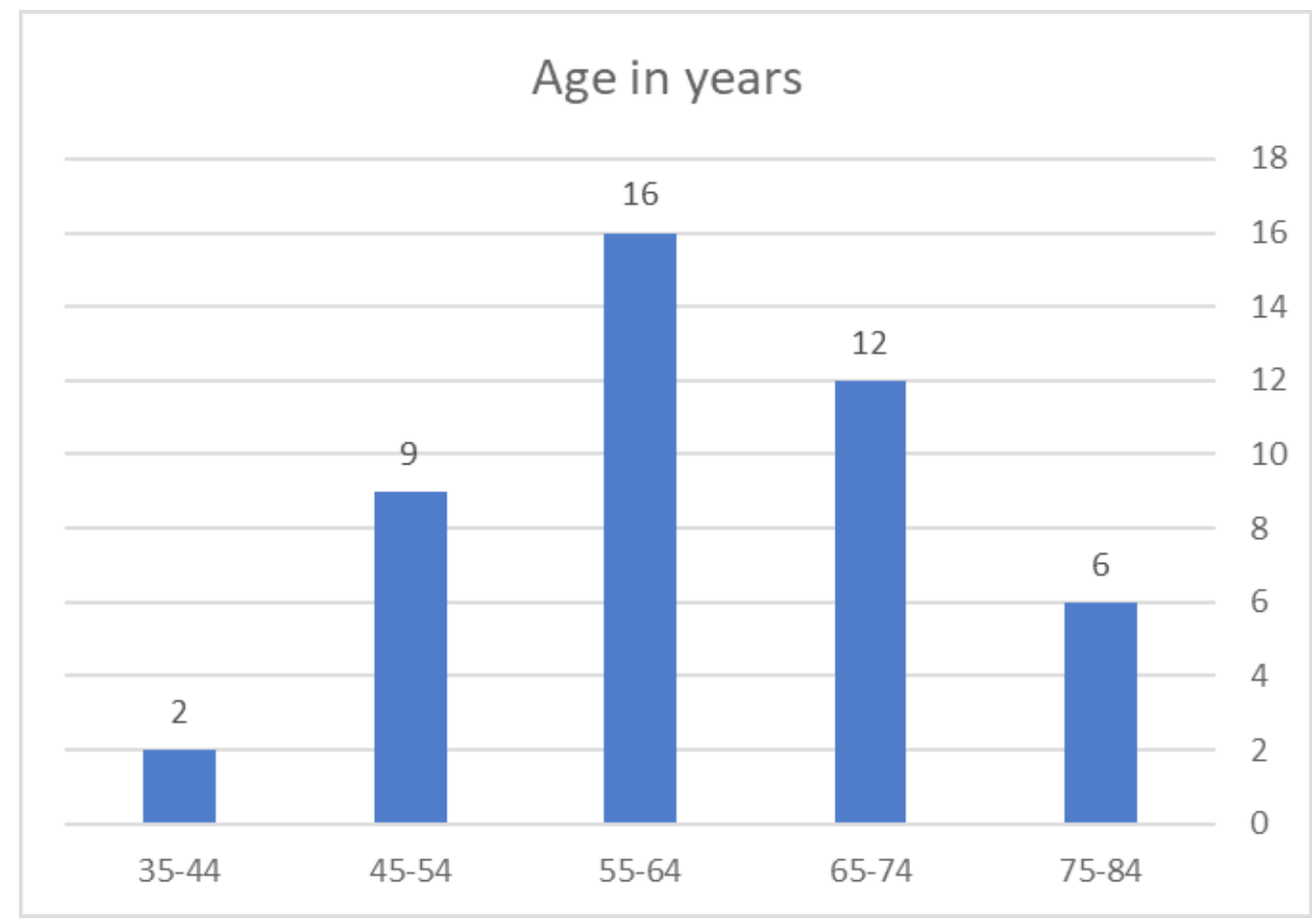

Figure (1): Age Demographic Distributions Among Patients with CA Bladder

According to this figure, the age distributions of CA Bladder patients in this study show a peak level in the age of 55-64 years and the least between 35-44 years age groups. The mean age of Bladder cancer in this study was 62 years, and it is near the mean age reported in a previous study done in Iraq which is 60 years ${ }^{[20-24]}$.

Table (1): Means Age \pm SD of Bladder Cancer Patients Compared to Control.

\begin{tabular}{|l|l|c|c|c|}
\hline & Subject & No. & Mean \pm SD & Range \\
\hline \multirow{4}{*}{ Age (years) } & Newly diagnosis & 19 & $59.28 \pm 11.26$ & $35-76$ \\
\cline { 2 - 5 } & Treatment and fallow up & 26 & $64.55 \pm 6.92$ & $52-84$ \\
\cline { 2 - 5 } & Control & 45 & $46.15 \pm 6.39$ & $31-67$ \\
\hline P.value & Newly diagnosis versus Treatment and fallow up (P.>0.05) \\
\hline
\end{tabular}

The number of male's patients in this study was 34 in the ages ranging between 35-80 with mean \pm SD (59.7 \pm 10.7$)$ while the female's number was 11 in the ages ranging between $48-84$ with mean $\pm \mathrm{SD}(63.1 \pm 10.0)$. The ratio of males to females in CA Bladder patients in this study represented to $4: 1$ proportion. The demographic distribution of male and female in this study, the male's patients have the greatest ratio than females, these ratios agrees with other studies done in Iraq which concluded to a similar ratio between male and female with CA bladder in his study. According to the history of Bladder cancer patients participated in this study all 30 male patient and 5 female patients was a heavy smoker for a long time. In the present study, the level of arginase in the urine of CA Bladder patients was evaluated in the two groups, the first group, G1 newly diagnosis 
$(94.6+17.2)$, and second group G2 treatment and followup (.91.2 \pm 13.6$)$,compared with normal patients control group (77.9 \pm 12.9$)$. In this study, urinary arginase was significantly higher in both groups of Bladder cancer patients G1 and G2 compared to normal control group of patients (CG) P value $<0.001$. In G1 (New Diagnosis) urinary arginase was negligible in contrast with G2 (Treatment and fellow up) P-value $>0.05$.

Table (2): The Mean \pm SD of Urinary Arginase (Arg) in CA Bladder Compared to Control

\begin{tabular}{|l|l|c|c|}
\hline Parameter & Subjects & No. & Mean \pm SD \\
\hline \multirow{3}{*}{ Urinary arginase } & Newly diagnosed & 19 & $94.6 \pm 17.2$ \\
\cline { 2 - 4 } & Treat. \& fallow up. & 26 & $91.2 \pm 13.6$ \\
\cline { 2 - 4 } & Control & 45 & $77.9 \pm 12.9$ \\
\hline \multirow{2}{*}{ P-value } & $\begin{array}{l}\text { Newly diagnosed versus Control group (P <0.001) } \\
\text { Treat. \& fallow up. versus Control group (P < 0.001) } \\
\text { Newly diagnosed versus Treat. \& fallow up (P }>0.05)\end{array}$ \\
\hline
\end{tabular}

Throughout this analysis, the amount of urinary arginase increases with an increase in the cancer stage, thereby increasing cell degradation and releasing more protein molecules in the urine, such as arginase. Hence, this enzyme may serve as a useful biological urinary marker in bladder cancer while also being an indication of the progression of bladder cancer. These findings of the previous research support report have showed that the amount of arginase in the different types of cancer increases as the tumor stage increases.

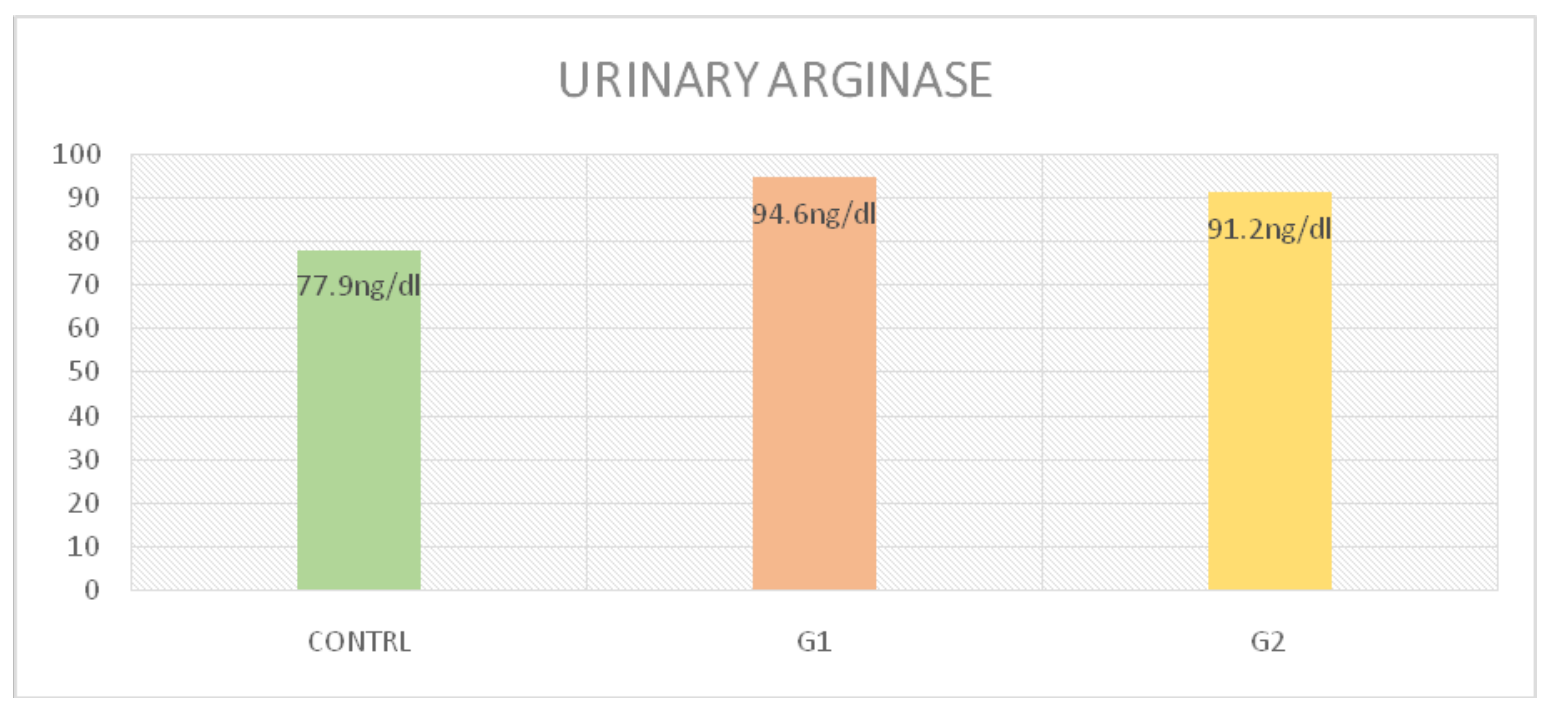

Figure(2): Arg in CA bladderand control groups

To extract sensitivity and specificity, Medcalc was used. Medcalc is a simple statistical program with full capabilities, as it contains all the functions and statistical tests, in addition to the lightness of the program and simple graphical interfaces from us, and also features great graphics ${ }^{[27]}$. This program was used to obtain the sensitivity and Specificity of Urinary Arginase that is managed through test schedules the results are as follows:
Table (3): Sensitivity and specificity of Urinary Arginase

\begin{tabular}{|l|l|c|}
\hline Abbreviations & Parameter & Value \\
\hline Sens. & Sensitivity & 92.59 \\
\hline Spec. & Specificity & 51.52 \\
\hline AUC & Area under the ROC curve & 0.716 \\
\hline P.Value & Probability value & 0.001 \\
\hline
\end{tabular}




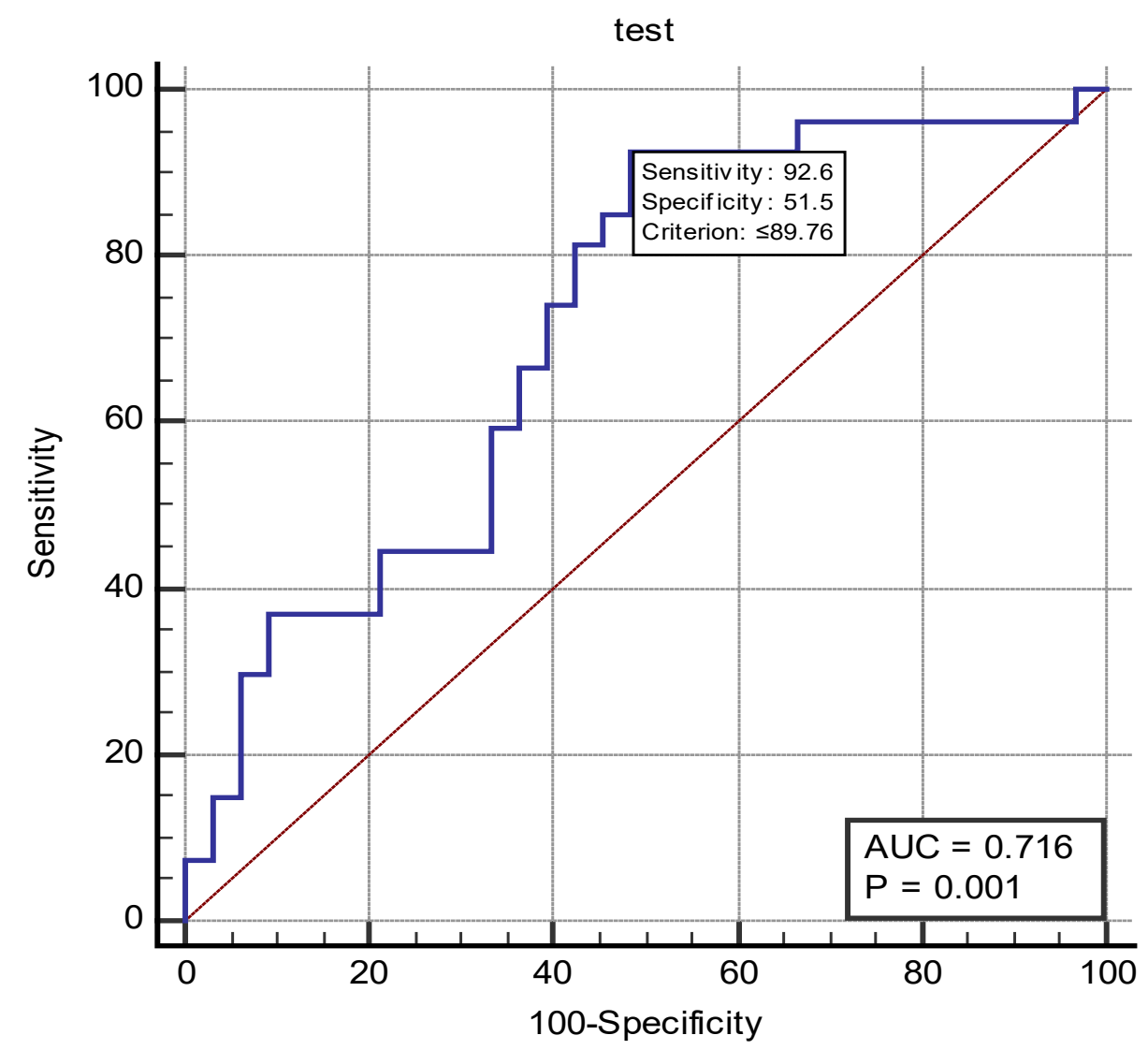

Figure (3): Sensitivity and Specificity of Urinary Arginase

\section{Conclusions}

CA bladder patients in Babylon province have high urinary arginase level comparing to normal subjects. Urinary arginase can be used as CA bladder protocol during the course of follow up for CA bladder patient and may help to minimize the need for an invasive procedure or radiation imaging. Therefore, please work on improving the sensitivity and specificity to test for Urinary Arginase so that it can be used as a protocol in the follow-up of bladder cancer patients.

Ethical Clearance: The Research Ethical Committee at scientific research by ethical approval of both $\mathrm{MOH}$ and MOHSER in Iraq.

\section{Conflict of Interest: None}

Funding: Self-funding

\section{References}

1. Miyazaki J, Nishiyama H. Epidemiology of urothelial carcinoma. International Journal of Urology. 2017 Oct;24(10):730-4.
2. Bajic P, Wolfe AJ, Gupta GN. The urinary microbiome: implications in bladder cancer pathogenesis and therapeutics. Urology. 2019 Apr 1;126:10-5.

3. Compérat E, Varinot J, Moroch J, EymeritMorin C, Brimo F. A practical guide to bladder cancer pathology. Nature Reviews Urology. 2018 Mar;15(3):143

4. Van Osch FH, Jochems SH, van Schooten FJ, Bryan RT, Zeegers MP. Significant role of lifetime cigarette smoking in worsening bladder cancer and upper tract urothelial carcinoma prognosis: a meta-analysis. The Journal of urology. 2016 Apr 1;195(4):872-9.

5. Santoni G, Morelli MB, Amantini C, Battelli N. Urinary markers in bladder cancer: an update. Frontiers in oncology. 2018 Sep 7;8:362.

6. Duenas $\mathrm{M}$, Martínez-Fernández $\mathrm{M}$, GarcíaEscudero R, Villacampa F, Marqués M, SaizLadera C, Duarte J, Martínez V, Gómez MJ, Martín ML, Fernández M. PIK3CA gene alterations in 
bladder cancer are frequent and associate with reduced recurrence in non-muscle invasive tumors. Molecular carcinogenesis. 2015 Jul;54(7):566-76.

7. Rodrigues DF. Volatile Organic Compounds for the Detection of Bladder Cancer: an In Vitro Metabolomic Approach.

8. Santoni G, Morelli MB, Amantini C, Battelli N. Urinary markers in bladder cancer: an update. Frontiers in oncology. 2018 Sep 7;8:362.

9. Marti Lindez AA. Arginase 2 as a metabolic immune checkpoint in anti-tumor immunity (Doctoral dissertation, University of Geneva).

10. Rath M, Müller I, Kropf P, Closs EI, Munder M. Metabolism via arginase or nitric oxide synthase: two competing arginine pathways in macrophages. Frontiers in immunology. 2014 Oct 27;5:532

11. S Clemente G, van Waarde A, F Antunes I, Dömling A, H Elsinga P. Arginase as a Potential Biomarker of Disease Progression: A Molecular Imaging Perspective. International Journal of Molecular Sciences. 2020 Jan;21(15):5291.

12. Banerjee B, Koner D, Lal P, Saha N. Unique mitochondrial localization of arginase 1 and 2 in hepatocytes of air-breathing walking catfish, Clariasbatrachus and their differential expression patterns under hyper-ammonia stress. Gene. 2017 Jul 30;622:13-22.

13. Sin YY, Ballantyne LL, Mukherjee K, Amand TS, Kyriakopoulou L, Schulze A, Funk CD. Inducible arginase 1 deficiency in mice leads to hyperargininemia and altered amino acid metabolism. Plos one. 2013 Nov 4;8(11):e80001.

14. Bhatta A, Yao L, Toque HA, Shatanawi A, Xu Z, Caldwell RB, Caldwell RW. Angiotensin IIinduced arterial thickening, fibrosis and stiffening involves elevated arginase function. PLoS One. 2015 Mar 25;10(3):e0121727.

15. Pernow J, Jung C. Arginase as a potential target in the treatment of cardiovascular disease: reversal of arginine steal?. Cardiovascular research. 2013 Jun 1;98(3):334-43.

16. Durante W. Role of arginase in vessel wall remodeling. Frontiers in immunology. 2013 May 13;4:111.

17. Caldwell RW, Rodriguez PC, Toque HA, Narayanan SP, Caldwell RB. Arginase: a multifaceted enzyme important in health and disease. Physiological reviews. 2018 Apr 1;98(2):641-65.

18. Munder M. Arginase: an emerging key player in the mammalian immune system. British journal of pharmacology. 2009 Oct;158(3):638-51.

19. Sakamoto S, Putalun W, Vimolmangkang S, Phoolcharoen W, Shoyama Y, Tanaka H, Morimoto S. Enzyme-linked immunosorbent assay for the quantitative/qualitative analysis of plant secondary metabolites. Journal of natural medicines. 2018 Jan 1;72(1):32-42.

20. Aldrich JO. Using IBM SPSS statistics: An interactive hands-on approach. Sage Publications; 2018 Aug 29.

21. Al-Baaj AF, Ewadh MJ, Abood MR. Total Glutathione Levels Correlation with Arginase in Serum \& Urine in CA Bladder Patients in Babylon Governorate. Journal of Pharmaceutical Sciences and Research. 2018 Oct 1;10(10):2578-81.

22. Hamzah H. Kzar, Abd Mena Y, Murad Manal M. Evaluation of Oxidative Stress Status: Total Antioxidant Ratio in Serum of Patients with Metastasis and Non-metastasis Lung Cancer in Babylon Province.ndian Journal of Forensic Medicine \& Toxicology. 2019, 13 (4): 832-835.

23. Joodi, M.R., Al-Hussainy, A.F.M.Lower urinary system injuries in association with previous repeated caesarian sections: Cross sectional study; Annals of Tropical Medicine and Public Health, 2020; 23 (1), S413.

24. Attarbashii AA. Urethrocystoscopic Features in Cases with Urinary Blandder Cancer in Mosul:" a Case Series Study". Tikrit Medical Journal. 2013;19(1): 12-15.

25. Ahmed,N.S.,Jasim,S.A., Majeed,A.A.Phylogenetic tree and submission of local enterobacter cloacae isolate from an urinary tract infection (UTI) of Iraq woman in NCBI, Biochemical and Cellular Archives, (2019) :19 (2), pp. 4003-4007.

26. Hussein YF. Regional Differences in Histological Features of Bladder Cancer Diagnosed in Post-War Iraq. Int J Cancer Epid \& Res. 2017;1:2-8.

27. Garber, C. (1998). MedCalc Software for Statistics in Medicine. MedCalc Software, Broekstraat 52, 9030 Mariakerke, Belgium, \$399.00. Clinical Chemistry, 44(6), 1370-1370. 